\title{
A NEW SIMPLIFIED ANALYTICAL DESIGN METHOD FOR STEEL AND COMPOSITE SWAY FRAMES
}

\section{Jean-François Demonceau ${ }_{a}$,Jean-Pierre Jaspart ${ }_{b}$}

a University of Liège, Chemin des Chevreuils, 1 B52/3 4000 Liège, Belgium, jfdemonceau@ulg.ac.be (corresponding author - Tel: +3243669358 - Fax: +3243669192)

b University of Liège, Chemin des Chevreuils, 1 B52/3 4000 Liège, Belgium, jeanpierre.jaspart@ulg.ac.be

\begin{abstract}
Eurocode 4 is the European design code for composite construction; in its so-called EN 19941-1 version, the design of "non-sway buildings" is mainly covered. As a result, EC4 focuses on the check of structural elements like beams, columns, slabs and joints. However, in the last years, the construction of taller buildings and larger industrial halls without wind bracing systems tends to make global instability a relevant failure mode, which is not well covered by Eurocode 4. Recently, intensive experimental, numerical and theoretical investigations have been carried out at Liège University. The latter aimed at improving the knowledge in the field of sway composite building frames and at developing appropriate design rules. The rotational behavior of the beam-to-column composite joints is one of the key aspects of the problem to which a special attention has been paid. This paper reflects investigations carried out at Liège University on this topic. In particular, an innovative simplified analytical method to predict the ultimate loading factor and the associated collapse mode of both steel and composite frames subjected to static loadings is presented.
\end{abstract}

Keywords: sway frames; new analytical design method; composite frames; steel frames 


\section{INTRODUCTION}

Most composite structures are laterally restrained by efficient bracing systems, such as concrete cores. This practice does not favor the use of composite structures. Indeed, once concrete construction companies are involved into major parts of a building, the reason for using composite structures for subsequent parts is often questionable.

Moment resisting frames offer a flexible solution to the user of the buildings, especially for the internal arrangement and the exploitation of the buildings. When sufficient stiffness and strength with regard to lateral forces are achieved, such frames offer a structural solution, which can resist lateral loads. In seismic regions, properly designed moment resisting frames are the best choice regarding the available ductility and the capacity to dissipate energy. This is stated in Eurocode 8 [1] devoted to earthquake engineering in which high values of the behavior factor are recommended for such frames.

These frames are prone to second-order effects; these effects have to be predicted carefully because they may govern the design. First investigations in this field have been carried out; in particular, the applicability of the wind-moment method to unbraced composite frames was first examined in a Ph.D thesis submitted at Nottingham University (Hensman, 1998 [2]). As far as the European codes are concerned, Eurocode 4 [3], which deals with composite constructions under static loading, contains mainly design procedures for non-sway composite buildings and gives design rules for composite slabs, beams, columns and joints. That is why a research project on global instability of composite sway frames was funded in 2000 by the European Community for Steel and Coal [4]. The objective of this project was to provide background information on the behavior of such frames under static and seismic loads and to provide simplified design rules. Liège University, as part of this project, has contributed to the conducted experimental, numerical and analytical investigations [5]. In particular, a simplified analytical method aiming at predicting the ultimate load factor of steel and composite sway 
frames have been developed and validated. The present paper describes the developed method in details.

A first section briefly describes the preliminary investigations which were requested prior to the development of the simplified analytical method. Then, the developed method is described and its validation through parametrical numerical studies is detailed.

\section{PRELIMINARY INVESTIGATIONS}

Before the development of the simplified analytical method, some preliminary investigations were conducted with the objective (i) to validate some useful analytical and numerical tools and (ii) to identify the particularities in the behavior of composite sway frames. These preliminary investigations are briefly summarized herein.

\subsection{Validation of useful analytical and numerical tools}

The behavioral response of the beam-to-column joints is known to significantly influence the global behavior of sway structures. Accordingly, experimental and analytical investigations devoted to the study of the behavior of composite joints were conducted. Through the performed investigations, the use of the so-called "component method", which is the method recommended in the Eurocodes for the characterization of steel and composite joints, was validated ([5] \& [6]). In particular, the component method was improved in order to be able to predict the response of composite joints subjected to "sagging" moment, situation not presently covered in the codes ([5] \& [7]) but which can appear in sway frames.

Then, a homemade finite element software, called FINELG, used for the prediction of the composite sway frame responses, was validated through a benchmark study (realized amongst European Institutions [4]) and through comparisons with the results of experimental tests performed on composite frames in two European laboratories [5]. 
The software FINELG is a finite element program able to follow the behavior of a structure under increasing external loading up to collapse or instability and even beyond, including geometrical non-linearities (using either the total, the up-dated or the corotational lagrangian formulation) and the material non-linearities (via the incremental plasticity theory (small strains)).

For the simulation of composite frames, 3 nodes plane beam elements with distributed plasticity are used (Figure 1) for the steel and the concrete elements. Node 1 and 3 present three degrees of freedom ( $u, v$ and $\theta$ - see Figure 1); node 2 only presents one degree of freedom $(u)$ which allows taking into account of an eventual relative displacement between the concrete and the steel profile (not taken into account in the studies presented herein).

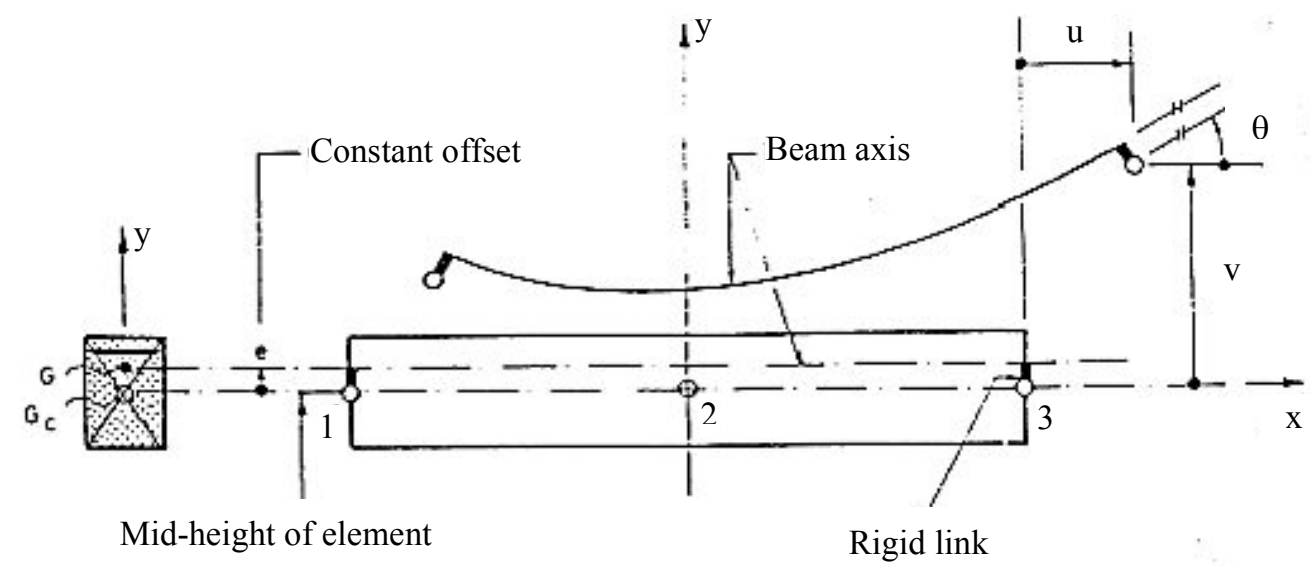

Figure 1. Plane beam finite element with three nodes used in FINELG for the composite frame modeling

For the validation of the software through comparisons to experimental tests, the actual properties for the materials were considered using an elastic-plastic behavior law with strain hardening for the steel elements and a parabolic law with tension stiffening for the concrete elements. At the end of these investigations, the ability of FINELG to accurately simulate the behavior of composite sway frames was demonstrated [5]; an example of a comparison between the numerical prediction obtained through FINELG and the experimental result 
obtained through a test performed in Bochum on a 2-bays - 2-storeys composite sway frame with semi-rigid and partial-strength joints [4] is illustrated in Figure 2.

Through the so-performed validations, the results obtained through FINELG for the prediction of the behavior of composite sway frames, including the joint behavior, may be considered as the results of reference reflecting the actual behavior of the frames; it is what has been assumed in the studies presented here after.

Composite frame tested in Bochum [4]
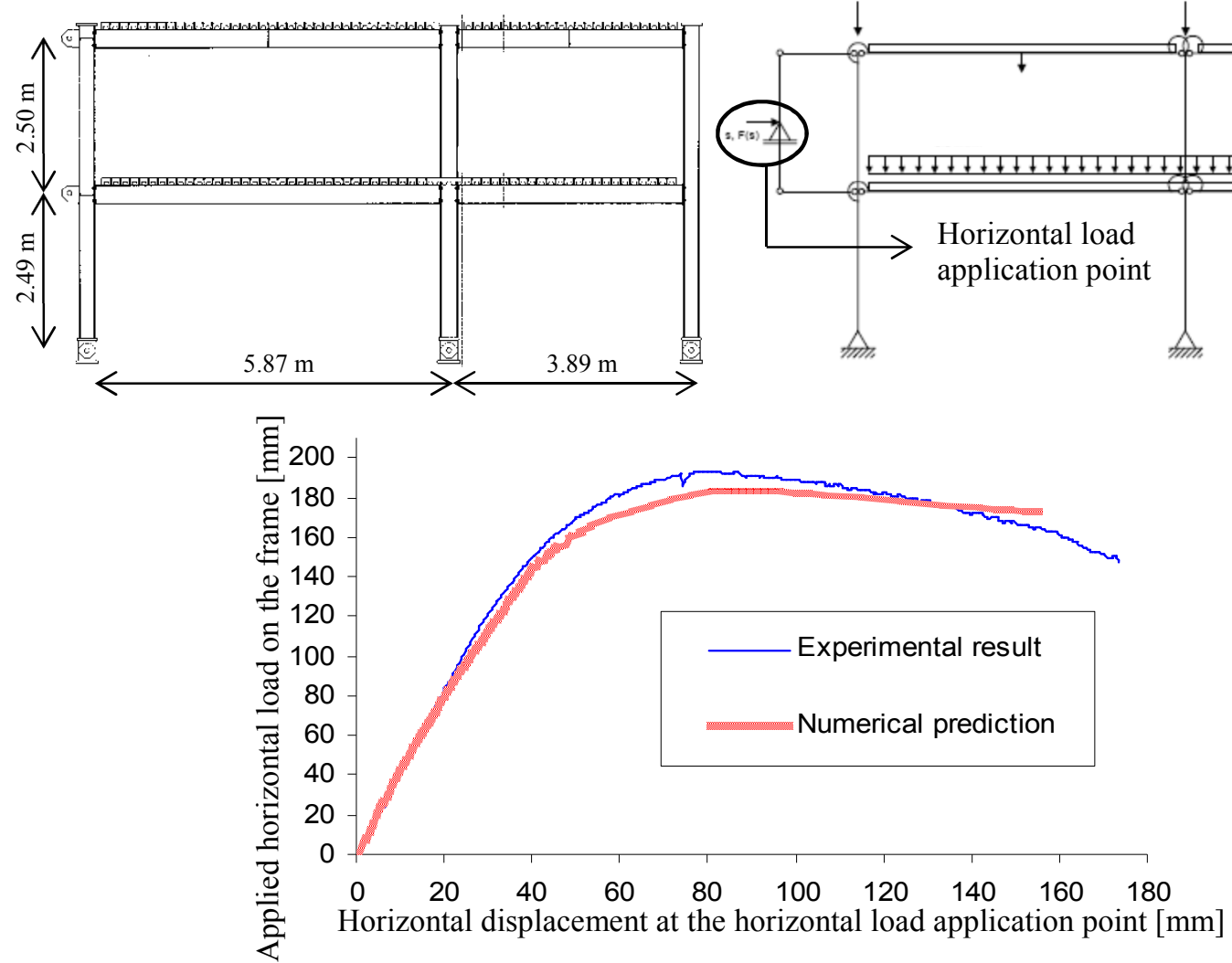

Loading and static scheme of the tested frame [4]

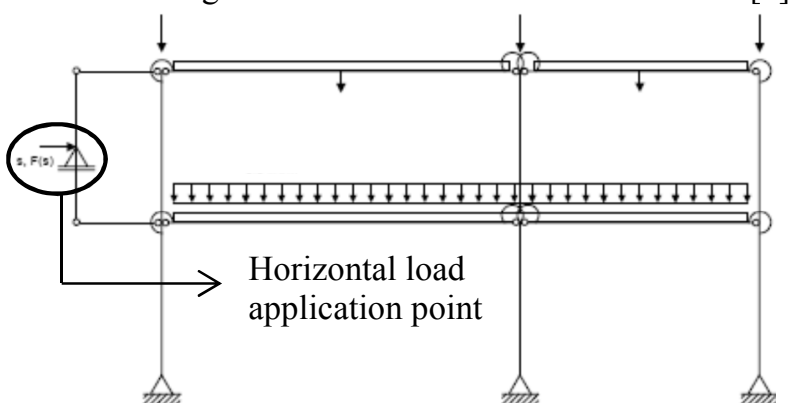

Figure 2. Bochum test - comparison between the numerical prediction and the experimental result [5]

\subsection{Identification of the particularities in the behavior of composite sway frames}

Composite sway structures present a specificity in comparison with steel ones: the concrete cracking. This phenomenon leads to an amplification of the lateral deflections and consequently to an amplification of the second-order effects, which reduces the ultimate resistance of the frames. In other words, for a same number of plastic hinges formed at a given load level in a steel frame and in a composite frame respectively, larger sway 
displacements are observed for the composite one as the concrete cracking which is initiated at the beginning of the loading affects the global stiffness of the composite frame. Numerical and analytical investigations were performed with the previously validated tools in order to characterize the behavior of composite sway frames under static loading ([8] and [9]). In particular, five composite sway frames extracted from actual or tested buildings were numerically studied. From these numerical studies, it was demonstrated that the general behavioral response of such structures to static vertical and horizontal loads is quite similar to the one exhibited by steel sway frames. Starting from this observation, the applicability to composite sway frames of two simplified analytical methods initially dedicated to steel ones was investigated: the "amplified sway moment method" and the "Merchant-Rankine approach" (respectively based on elastic and plastic design philosophies).

\subsubsection{Amplified sway moment method}

This simplified analytical method is proposed in Eurocode 3 [11] dealing with steel structures. In this method, first-order linear elastic analyses are first carried out; then, the resulting internal forces are amplified by a "sway factor" so as to ascertain for second-order sway effects. Finally, the design load resistance of the frame may be derived by computing the load at which a first plastic hinge develops in the frame (i.e. the elastic load factor $\lambda_{e}$ is derived).

The steps to be crossed when applying this elastic design procedure are as follows:

- A first-order elastic analysis is performed on the frame fitted with horizontal supports at the floor levels (Figure 3.A); it results in a distribution of bending moments in the frame and reactions at the horizontal supports.

- Then, a second first-order elastic analysis is conducted on the initial frame subjected to the sole horizontal reactions obtained in the first step (Figure 3.B); the resulting bending moments are the so-called "sway moments". 
Approximate values of the "actual" second-order moments result from the summing up of the moments obtained respectively in the two frame analyses, after having amplified the sole sway moments by means of the sway factor:

$$
\frac{1}{1-\frac{V_{E d}}{V_{c r}}}
$$

where $V_{E d}$ is the design vertical applied load and $V_{c r}$ is the lowest elastic critical load associated to a global sway instability.

The maximum elastic resistance of the frame is reached as soon as a first plastic hinge forms in the frame.
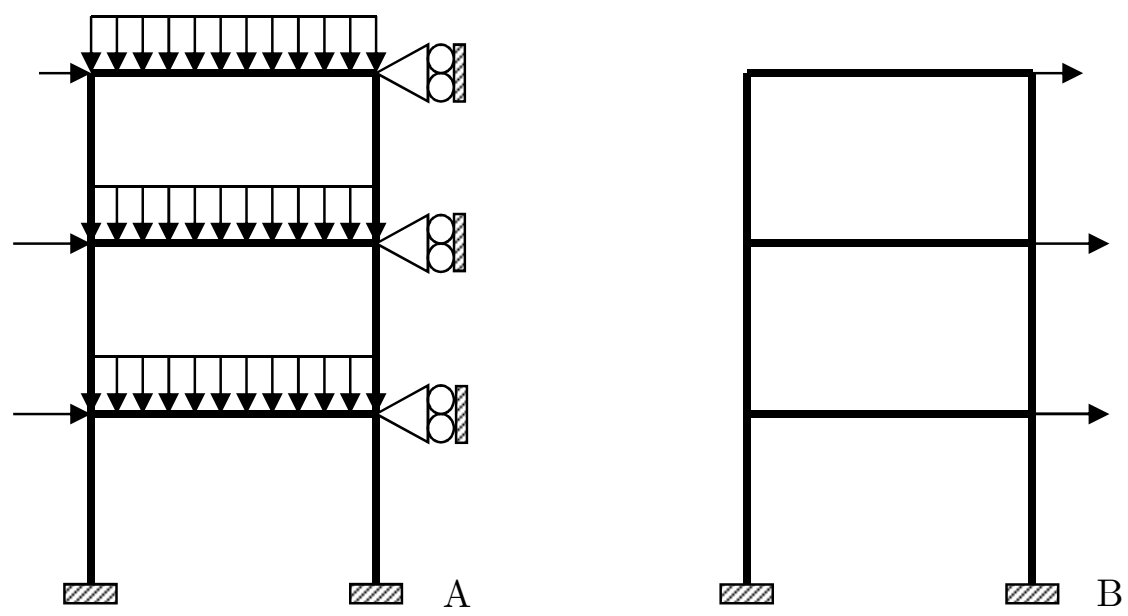

Figure 3. Static schemes used for the amplified sway moment method For this method, it was demonstrated that a good accuracy is obtained when applied to sway composite structures by comparing the elastic load factor $\lambda_{e}$ predicted through the "amplified sway moment method" and through FINELG for the five previously mentioned actual sway buildings. For these five cases, a maximum difference of $5 \%$ on the predicted $\lambda_{e}$ has been observed. Accordingly, this method can be recommended for this type of structures. 


\subsubsection{Merchant-Rankine approach}

The "Merchant-Rankine approach" allows predicting the ultimate load factor of a structure, $\lambda_{u}$, as a function of the plastic load factor, $\lambda_{p}$, obtained through a first-order rigid-plastic analysis and the critical load factor, $\lambda_{c r}$, obtained through a critical analysis, as follows:

$$
\frac{1}{\lambda_{u}}=\frac{1}{\lambda_{c r}}+\frac{1}{\lambda_{p l}}
$$

For this approach, it was shown that the conclusions that were drawn concerning the accuracy of this method for steel sway structures (Maquoi and Jaspart 2001 [10]) are still valid for composite sway structures, i.e the method is safe when $\lambda_{p}$ is associated to a beam plastic mechanism, adequate when $\lambda_{p}$ is associated to a combined plastic mechanism and unsafe when $\lambda_{p}$ is associated to a panel plastic mechanism. Moreover, the nature of the plastic mechanism considered in the Merchant-Rankine approach does not always correspond to the one occurring in the frame at failure (computed through a non-linear analysis), i.e. when $\lambda_{u}$ is reached. This phenomenon is due to the second-order effects which differently influence the yielding of the structure according to the nature of the considered plastic mechanism. For instance, if $\lambda_{p}$ is associated to a beam plastic mechanism, the ultimate load factor $\lambda_{u}$ may be associated to the development of a panel plastic mechanism as the latter is strongly influenced by the geometrical second-order effects while the beam mechanism is not [5].

According to these observations, it was decided to develop a new simplified analytical method able to predict the ultimate load factor and its associated collapse mode accurately. This method is presented in the following section.

\section{DEVELOPED SIMPLIFIED ANALYTICAL METHOD}

The proposed procedure is based on three formulas, one for each possible type of plastic mechanisms (i.e. beam, panel and combined plastic mechanisms): 
- $\operatorname{Formula1}\left(\lambda_{p, \text { beam }}, \lambda_{c r}\right) \rightarrow \lambda_{u, \text { beam }}$

- Formula2 $\left(\lambda_{\text {p,panel }}, \lambda_{c r}\right) \rightarrow \lambda_{u, \text { panel }}$

- $\operatorname{Formula3}\left(\lambda_{p, \text { combined, }} \lambda_{c r}\right) \rightarrow \lambda_{u, \text { combined }}$

Three ultimate load factors are then predicted from these formulas and the smallest one is considered as the ultimate load factor of the studied frame: $\lambda_{u}=\min \left(\lambda_{u, \text { beam }}, \lambda_{u \text {,panel }}\right.$, $\left.\lambda_{u, \text { combined }}\right)$.

These new formulas could have been derived from the Merchant-Rankine one. In fact, the actual Merchant-Rankine formula could be used as "Formula3" as it was demonstrated in [10] and in [5] that it gives satisfactory results when the first-order rigid-plastic mechanism of the frame is a combined one. Nevertheless, it was chosen to develop these formulas from the Ayrton-Perry formulation (see Table 1), which is already used in the Eurocodes to deal with the member instability phenomena (plane buckling, lateral buckling and lateral torsional buckling). This proposal is in agreement with the recommendation of the last draft of Eurocode 3 [11] where it is stated that such formulation should be used to verify "the resistance to lateral and lateral torsional buckling for structural components such as single members (built-up or not, uniform or not, with complex support conditions or not) or plane frames or subframes composed of such members which are subject to compression and/or mono-axial bending in the plane..." (§ 6.3 .4 (1) of Eurocode 3, Part 1-1[11]). A great advantage is that the Ayrton-Perry formulation implicitly allows respecting the limit conditions which are: (i) when $\lambda_{c r}$ is very high, no instability phenomena appears and the failure occurs through the appearance of a plastic mechanism $\left(\lambda_{u} \rightarrow \lambda_{p}\right)$ and (ii) when $\lambda_{p}$ is very high, no yielding appears in the frame and the failure occurs through an instability phenomenon $\left(\lambda_{u} \rightarrow \lambda_{c r}\right)$. 
Table 1. From the Ayrton-Perry formulation to the formulas to be included in the new simplified analytical design method

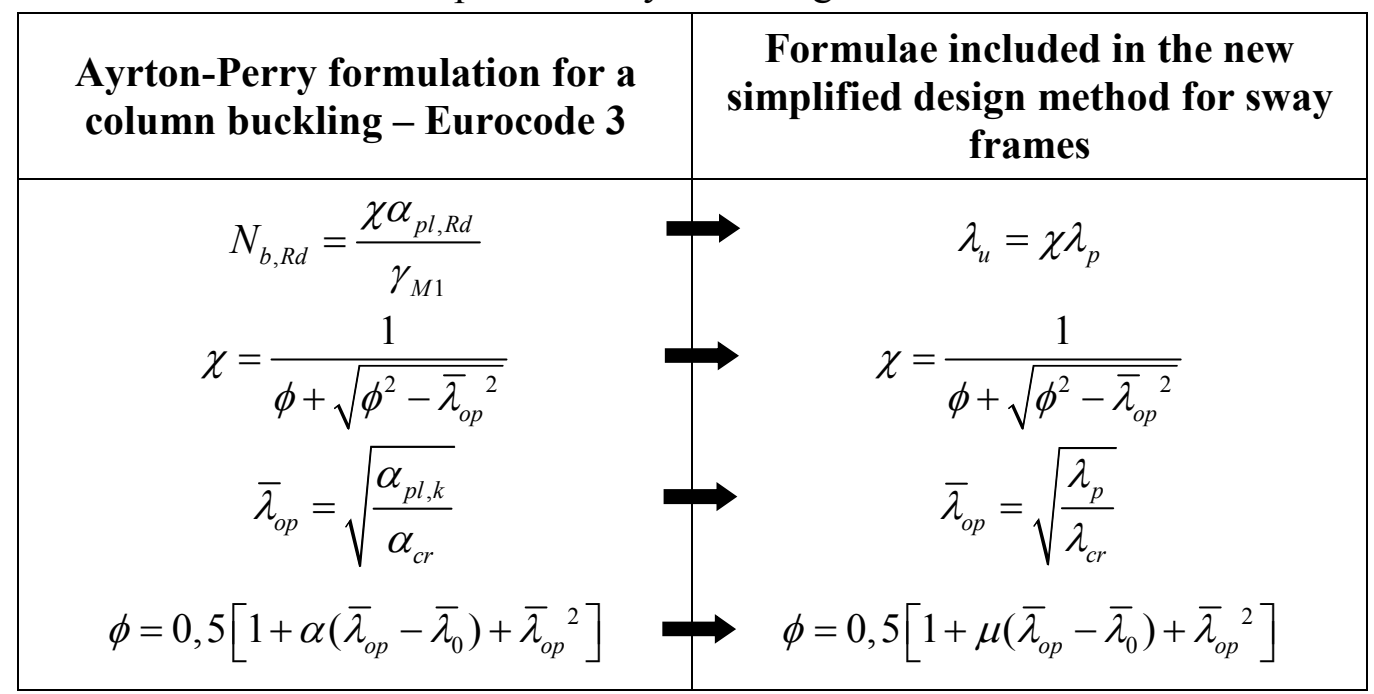

Within the formulation reported in Table 1:

- $\quad N_{b, R d}$ corresponds to the buckling design resistance of a column;

- $\quad \alpha_{p l, R d}$ and $\alpha_{p l, k}$ are respectively the design and the characteristic plastic resistances of the considered column;

- $\quad \alpha_{c r}$ is the critical load of the considered column.

- $\quad \chi$ is called the reduction factor;

- $\quad \bar{\lambda}_{o p}$ is the non-dimensional relative slenderness and;

- the parameter $\bar{\lambda}_{0}$ represents the length of the plateau where $\chi$ is equal to 1 in a $\bar{\lambda}_{o p}-\chi$ graph (see Figure 4). For $\bar{\lambda}<\bar{\lambda}_{0}$, the ultimate resistance is assumed to be equal to the plastic resistance and, accordingly, the influence of the second-order effects is neglected. As neither strain hardening nor cladding effects are considered within the presented study, the plateau length is taken equal to 0 as it is in the Merchant-Rankine approach. 
So, to develop this new method, only the parameter $\mu$ had to be determined. This parameter is used to take the second order effects implicitly into account within the developed procedure. In fact it influences the shape of the curve presented in Figure 4: the higher $\mu$ is, the smaller the reduction factor $\chi$ and, accordingly, the smaller the predicted $\lambda_{u}$ are.

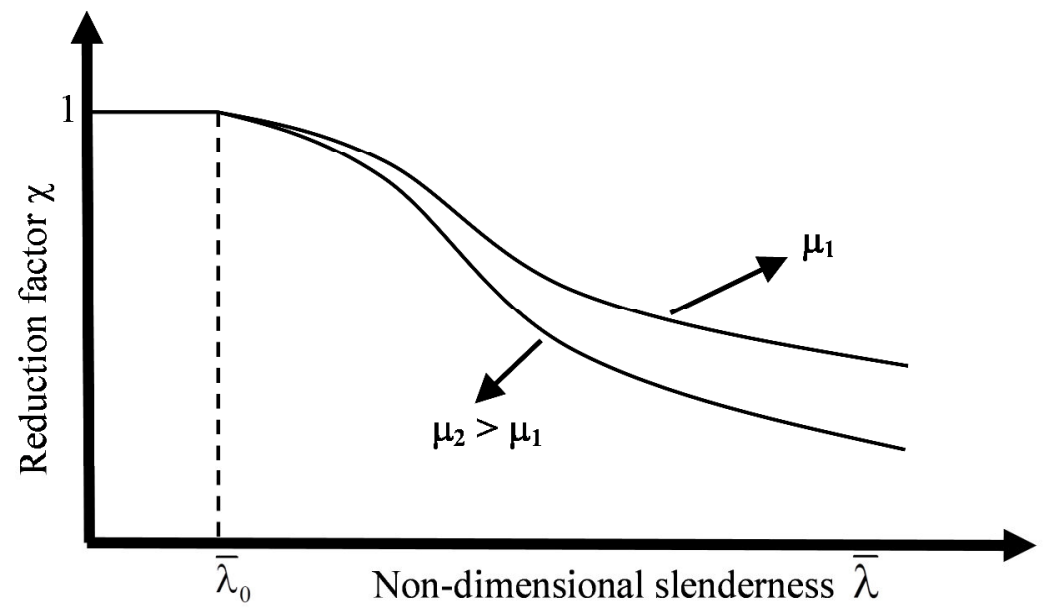

Figure 4. Example of "Ayrton-Perry" curves

Three values of the parameter $\mu$ had to be calibrated, one for each type of plastic mechanism (i.e. $\mu_{\text {beam }}$ for the beam plastic mechanism, $\mu_{\text {combined }}$ for the combined plastic mechanism and $\mu_{\text {panel }}$ for the panel plastic mechanism) as each one is influenced differently by the second order effects; accordingly, this results in the definition of three $\bar{\lambda}_{o p}-\chi$ curves (one for each type of plastic mechanism). These values have been calibrated through parametrical studies presented in the next section. At the end of this calibration, it is intended to obtain a higher value of $\mu$ for the panel plastic mechanism than the one for the combined plastic mechanism and the latter higher than the one for the beam plastic mechanism $\left(\mu_{\text {panel }}>\mu_{\text {combined }}>\mu_{\text {beam }}\right)$ as the influence of the second order effects is more important for the panel plastic mechanism than for the combined one and is not significant for the beam plastic mechanism [5].

As the same accuracy problems are met with the Merchant-Rankine approach for steel and composite sway frames, the proposed method has been developed for both types of frames. 
The calibration of the coefficient $\mu$ and the validation of the developed method are presented in the following section.

\section{CALIBRATION AND VALIDATION OF THE DEVELOPED METHOD}

The calibration of the coefficient $\mu$ and the validation of the developed method are performed through parametrical studies on steel and composite frames. The predictions obtained through the analytical method are compared to numerical predictions obtained through full non-linear analyses (realized with the previously validated software FINELG), considered as the "reference" results.

\subsection{Parametrical study on steel sway frames}

\subsubsection{Studied steel frames}

Within this study, four types of 2-D simple frames have been investigated (Figure 5); in total, 181 frames have been analyzed.

The beams and the columns are steel hot-rolled profiles of class 1 (i.e. with cross-sections allowing developing their plastic resistant moment and exhibiting a sufficient ductility to develop a full plastic mechanism in the frame); they are bent around their major axis. The steel material is modeled with an elastic-plastic behavior law for the non-linear analyses (neglecting the strain hardening effect as allowed in [11]).

The beam-to-column joints are classified as partial-strength and semi-rigid ones with a sufficient ductility to develop plastic hinges and to allow plastic analyses; they are modeled with rotational springs having an elastic-plastic behavior law. The column base joints are assumed to be rigid and fully resistant. The properties of the frames have been defined so as to cover the three types of plastic mechanisms, i.e. beam, combined and panel plastic mechanisms (obtained through first-order rigid-plastic analyses) with each type of structure and to obtain different types of collapse modes (plastic mechanisms or instability) through the full non-linear analyses. The parameters which have been modified within these frames are: 
- the height of the columns (from $4 \mathrm{~m}$ to $10 \mathrm{~m}$ );

- the properties of the joints (i.e. their stiffness and their resistance);

- the beam cross sections (IPE550 or IPE600);

- the column cross sections (HEA300 or HEB300) and;

- the applied loads.
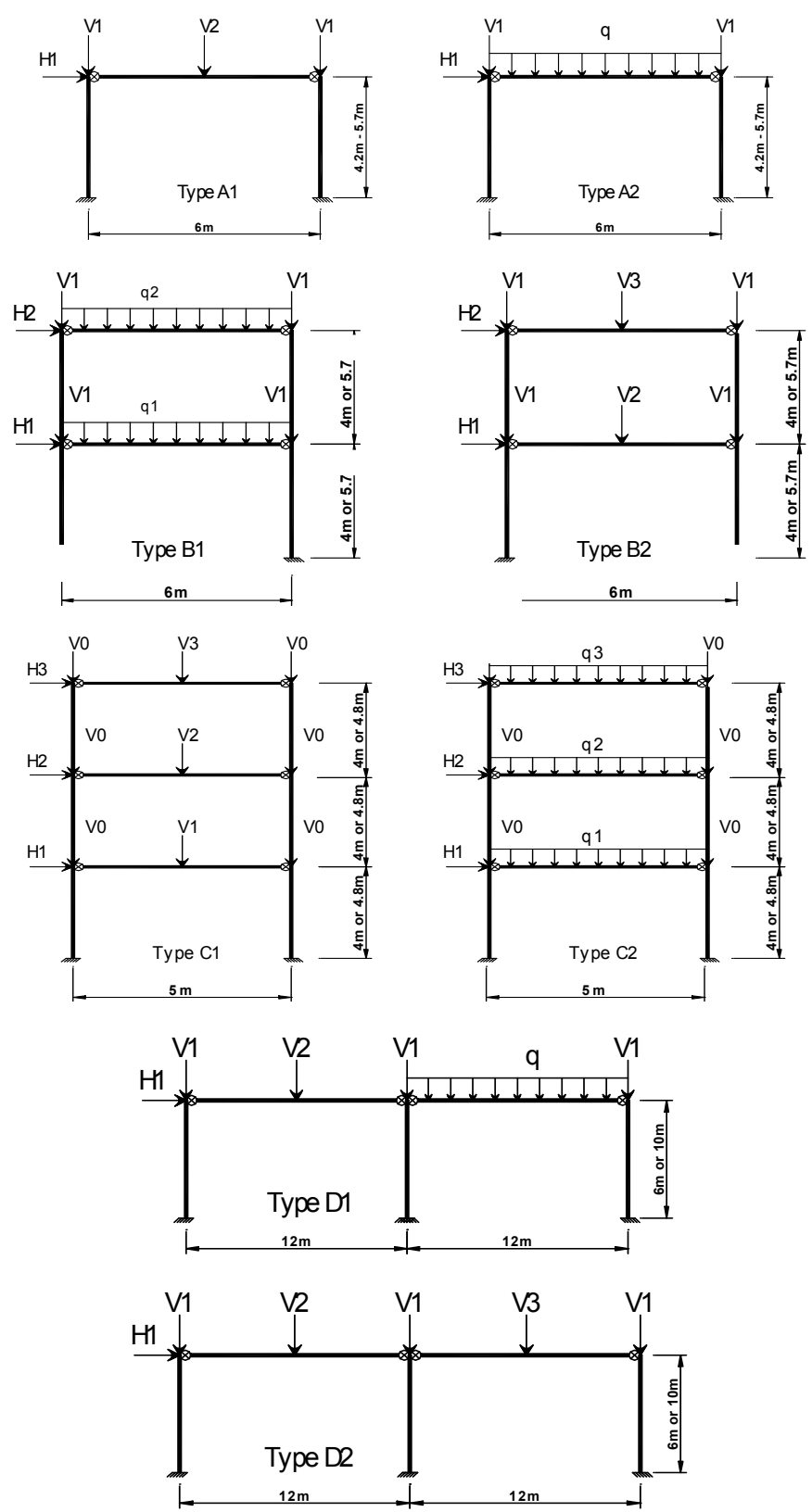

Figure 5. Studied steel frames - Types A, B, C \& D

The analyses which have been performed are:

- Critical elastic analyses $\left(\lambda_{c r}\right)$; 
- First-order rigid-plastic analyses (computation of the three plastic load factors, i.e. $\lambda_{p, \text { beam }}, \lambda_{p, \text { combined }}$ and $\left.\lambda_{p, \text { panel }}\right)$

- Full non-linear analyses $\left(\lambda_{u}\right)$.

For the computation of $\lambda_{c r}$ and $\lambda_{u}$, the software FINELG was used. As recommended in Eurocode 3 [11], an initial deformation has been introduced in the computation. The shape of the considered initial deformation corresponds to the first global instability mode obtained through the critical elastic analysis (which is in agreement with the Eurocode recommendations). This permits the introduction of a global initial deformation (to initiate $P$ $\Delta$ effects) and local initial deformations for the members (to initiate $P$ - $\delta$ effects) at the same time. For the computation of the plastic load factors, a software (based on an Excel sheet and Visual Basic modules) has been developed and validated through comparisons to numerical results. For the computation of the plastic load factors, the M-N interaction in the columns is taken into account using formulas permitting a very accurate analytical prediction of the actual M-N interaction curve for a double-T cross section.

\subsubsection{Parametric study results}

For each frame, the results obtained with the new method and with the Merchant-Rankine method are compared to the results of numerical non-linear analyses considered as the "reference" ones. The investigated frames were defined so as to cover a wide range of $\lambda_{p} / \lambda_{c r}$ values (from 0,09 to 0,61 ), $\lambda_{p}$ being the minimum value of the three plastic load factors $\lambda_{p, \text { beam }}, \lambda_{p, \text { combined }}$ and $\lambda_{p, \text { panel. }}$

The three values of $\mu$, i.e. $\mu_{\text {beam }}, \mu_{\text {combined }}$ and $\mu_{\text {panel }}$, reported here below have been chosen so as to minimize the difference between the values of $\lambda_{u}$ predicted using respectively the new method and the numerical analysis, without using specific calibration process:

- $\quad \mu_{\text {beam }}=0,070$ 
- $\quad \mu_{\text {combined }}=0,290$ and;

- $\quad \mu_{\text {panel }}=0,596$.

An improvement of the recommended values would consist in performing a probabilistic study to derive values of $\mu$ which would satisfy the criteria of the semi-probabilistic approach on which the Eurocodes are founded: this constitutes a perspective of the presented study.

The comparison between the values of $\lambda_{u}$ obtained through the analytical methods (the new one and the Merchant-Rankine approach) and the numerical simulations is given in Figure 6 and Figure 7 for all the frames.
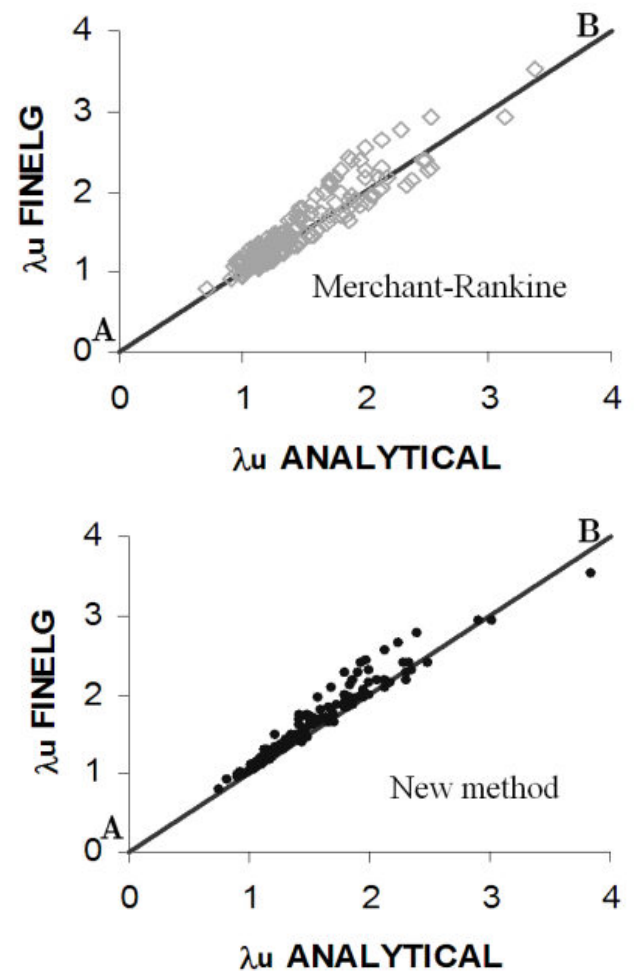

Figure 6. Comparison between the analytical and the numerical results for the prediction of $\lambda_{u}$ (all the investigated steel frames) 


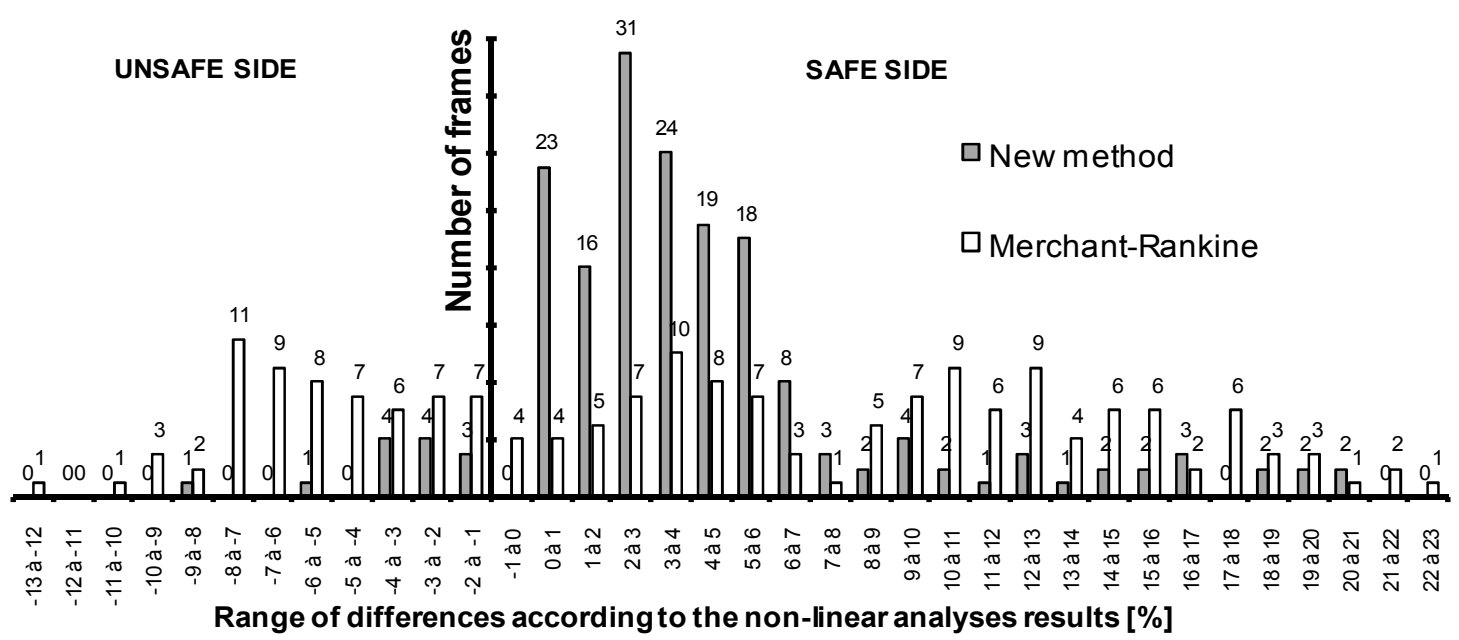

Figure 7. Evaluation of the accuracy of the analytical methods (all the investigated steel frames)

In Figure 6, the abscissa represents the analytically predicted values of $\lambda_{u}$ while the ordinate, the numerically computed ones. If the analytical methods were perfectly accurate, all the points of the figures would be exactly on line "AB", i.e. the analytical prediction would be equal to the numerical computation results. So, the more accurate the analytical method is, the closer to line "AB" the points are. Also, all the points which are in the upper zone of the graph with respect to line "AB" correspond to cases where the analytical method underestimates the ultimate load factors (i.e. "safe side" of the graph) while the points in the lower zone correspond to cases where the analytical method overestimates the ultimate load factors (i.e. "unsafe side" of the graph). From Figure 6, it can be observed that the new method gives more accurate results than the Merchant-Rankine approach; indeed, the points obtained with the new method are closer to line "AB" than the ones obtained with the Merchant-Rankine approach. Also, more points are on the "unsafe side" of the graph with the Merchant-Rankine approach than with the new method; indeed, the Merchant-Rankine approach is unsafe for 66 cases (i.e. $36 \%$ of the investigated frames) while the new method is unsafe for only 13 cases (i.e. $7 \%$ of the investigated frames). 
These observations are confirmed by the graph of Figure 7. The latter represents the number of frames which are included in given ranges of differences between the analytical predictions and the numerical results (expressed in \%). For instance, it can be seen on this graph that the number of frames for which the difference between the analytically predicted $\lambda_{u}$ and the numerically computed $\lambda_{u}$ is included in the range $[0 \% ; 1 \%]$ is equal to 23 with the new method and to 4 with the Merchant-Rankine approach. From Figure 7, it can be observed that the number of frames for which the differences on the value of $\lambda_{u}$ is between $0 \%$ and $10 \%$ is equal to 148 with the new method (i.e. $81,8 \%$ of the frames) and to 57 with the MerchantRankine approach (i.e. $31,5 \%$ of the frames), which confirms the better accuracy of the proposed method.

Also, as previously mentioned, the collapse mode associated to the ultimate load factor $\lambda_{u}$ does not necessarily correspond to the one associated to the plastic load factor $\lambda_{p}$; this reflects the situation of 112 of the investigated frames. It is interesting to underline that, with the new method, the type of plastic mechanism associated to the minimum value of $\lambda_{u}$ corresponds to the one appearing in the fully non-linear numerical analysis for $93 \%$ of the investigated frames.

In the presented results, the Merchant-Rankine approach is applied to all the frames with values of the $\lambda_{p}$ to $\lambda_{c r}$ ratio from 0,09 to 0,61 although it is recommended to apply this approach to structures with this ratio between 0,1 and 0,25 . If only the frames respecting this condition are considered (which is the case for 133 of the investigated structures), the previous observations are still valid; in particular:

- Only 4 unsafe situations (i.e. $3 \%$ of the considered frames) are obtained with the new method against 45 (i.e. $34 \%$ of the considered frames) with the Merchant-Rankine approach. 
- The number of frames for which the differences on the value of $\lambda_{u}$ is between $0 \%$ and $10 \%$ is now equal to 123 with the new method (i.e. $92,5 \%$ of the considered frames) and to 47 with the Merchant-Rankine approach (i.e. 35,3\% of the considered frames), which confirms the better accuracy of the proposed method.

- The type of plastic mechanism associated to the minimum value of $\lambda_{u}$ obtained with the proposed new method corresponds to the one appearing in the full non-linear numerical analysis for $93 \%$ of the investigated frames.

\subsection{Parametric study on composite sway frames}

\subsubsection{Studied composite frames}

Within this study, three types of 2-D simple frames have been investigated (Figure 8); in total, 199 frames have been studied. Different types of structural elements are met within the investigated frames as described here below:

- Two types of composite beam configurations bent around their major axis:

○ upper hot-rolled profile flange fully connected to a concrete slab or;

○ upper hot-rolled profile flange fully connected to a composite slab.

- Two types of columns bent around their major axis:

○ steel hot-rolled profiles or;

○ partially encased steel hot-rolled profiles.

- The beam-to-column composite joints are rigid or semi-rigid ones and full-strength or partial-strength ones; the column bases are assumed to be rigid and fully resistant. The beam-to-column joints are assumed to have sufficient ductility to develop plastic hinges and to allow a plastic analysis. 

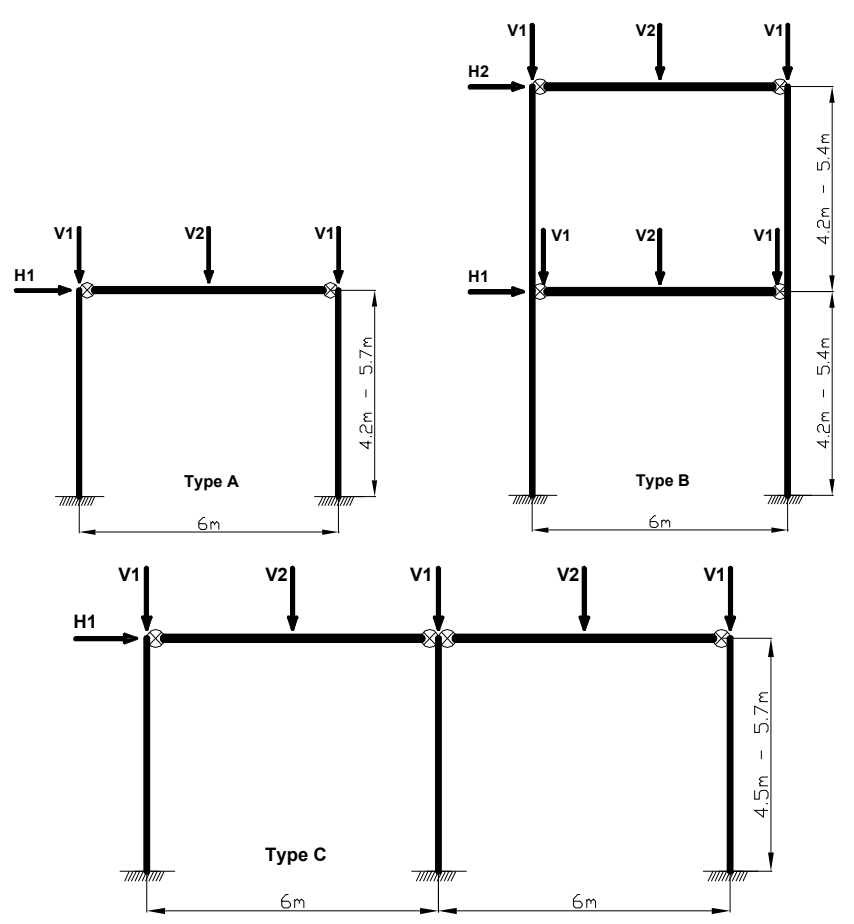

Figure 8. Studied composite frames - Type A, B and C

For the numerical simulations, the steel material and the joint behavior are modeled with an elastic-perfectly plastic bilinear law (neglecting the strain hardening effect as allowed in [11]). For the concrete material, a parabolic behavior law with account of tension stiffening is used.

As for the parametric study performed on the steel frames, the properties of the frames have been defined so as to cover the three types of plastic mechanisms, i.e. beam, combined and panel plastic mechanisms (obtained through first-order rigid-plastic analyses) for each type of structure and to obtain different types of collapse modes (plastic mechanisms or instability) through the full non-linear analyses. The parameters which have been modified within these frames are:

- the type of structural elements (as mentioned previously);

- the height of the columns $(4,2 \mathrm{~m}$ to $5,7 \mathrm{~m})$;

- the properties of the joints (i.e. their stiffness and their resistance);

- the beam and column cross sections and;

- the applied loads. 
For the computation of $\lambda_{c r}$ and $\lambda_{u}$, the software FINELG has been used. As recommended in Eurocode 4 [3], an initial deformation has been introduced in the computation. Also, as for the steel frames, the shape of the considered initial deformation corresponds to the first global instability mode obtained through the critical elastic analysis. For the computation of the plastic load factors, a software based on an Excel sheet has been developed and validated through comparisons to numerical results. For the computation of the plastic load factors, the $\mathrm{M}-\mathrm{N}$ interaction in the columns has been taken into account.

\subsubsection{Parametrical study results}

The investigated frames were defined so as to cover a wide range of $\lambda_{p} / \lambda_{c r}$ values (from 0,05 to 0,31$)$. The three values of $\mu$, i.e. $\mu_{\text {beam }}, \mu_{\text {combined }}$ and $\mu_{\text {panel }}$, calibrated so as to minimize the difference between the values of $\lambda_{\mathrm{u}}$ predicted using respectively the new method and the numerical analysis (as for the steel frames) are the following ones:

$$
\begin{aligned}
& -\quad \mu_{\text {beam }}=0,020 ; \\
& -\quad \mu_{\text {combined }}=0,420 \text { and; } \\
& -\quad \mu_{\text {panel }}=0,700 .
\end{aligned}
$$

It can be observed that these coefficients are higher than the ones calibrated for the steel structures (except for the values corresponding to the beam plastic mechanism which are very close), which means that, for a composite structure and a steel structure with the same value of $\lambda_{c r}$ and the same values of plastic load factors $\lambda_{p, \text { beam }}, \lambda_{p, \text { combined }}$ and $\lambda_{p, p a n e l}$, the ultimate load factor $\lambda_{u}$ obtained through the new method would be smaller for the composite structure than for the steel one.

This observation is in line with the remark on the effect of concrete cracking reported previously; this phenomenon leads to an amplification of the lateral deflections and, 
consequently, to an amplification of the second-order effects, which reduces the ultimate resistance of the frames. In other words, for a same number of plastic hinges formed at a given load level in a steel frame and in a composite frame, respectively, larger sway displacements are observed for the composite one. So, this particularity is reflected within the developed method through the " $\mu$ " values which are higher for composite sway frames than for the steel ones. The fact that the $\mu$ factors associated to the beam plastic mechanism are very close can be explained by the small influence of the second order effects on this type of collapse mode.

The comparison between the values of $\lambda_{u}$ obtained through the analytical methods (the new one and the Merchant-Rankine approach) and the numerical simulations is given in Figure 9 and Figure 10 for all the frames.
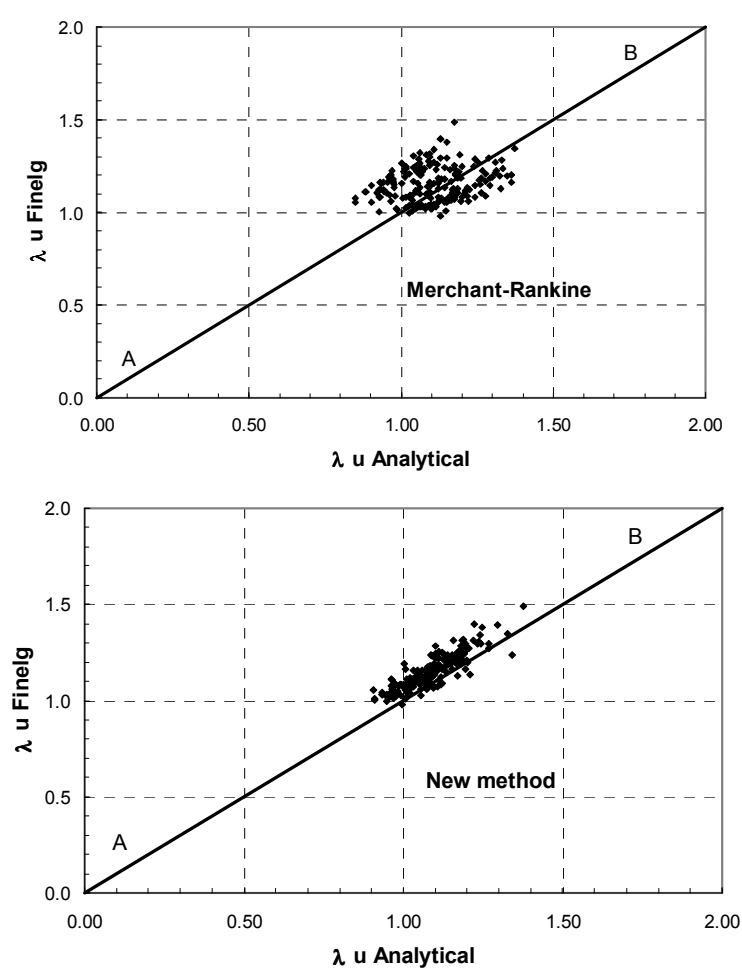

Figure 9. Comparison between the analytical and the numerical results for the prediction of $\lambda_{u}$ (all the investigated composite frames) 


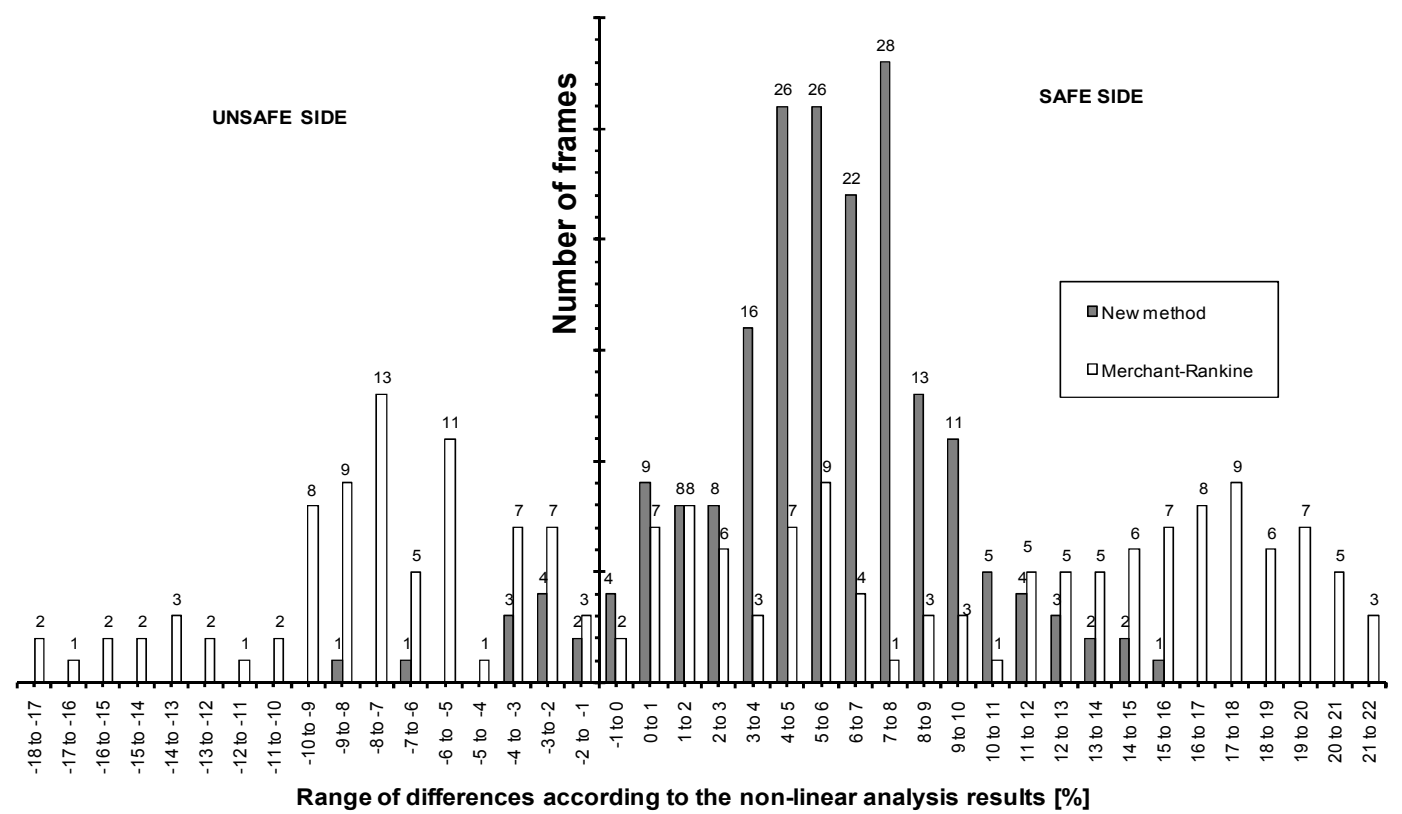

Figure 10. Evaluation of the accuracy of the analytical methods (all the investigated composite frames)

From Figure 9, it can be observed, as for the steel sway frames, that the new method gives more accurate results than the Merchant-Rankine approach; also, more points are on the "unsafe side" of the graph with the Merchant-Rankine approach than with the new method (the Merchant-Rankine approach is unsafe for 81 cases, i.e. $40,7 \%$ of the investigated frames, while the new method is unsafe for only 15 cases, i.e. 7,5\% of the investigated frames).

From Figure 10, it can be observed that the number of frames for which the difference between the analytically predicted values of $\lambda_{u}$ and the numerical ones is between $0 \%$ and 10 $\%$ is equal to 167 with the new method (i.e. $83,9 \%$ of the frames) and to 51 with the Merchant-Rankine approach (i.e. $25,6 \%$ of the frames), which confirms the better accuracy of the proposed method.

Also, among the investigated composite frames, there are cases (38 in total, i.e. $19,1 \%$ of the investigated composite frames) where the collapse mode associated to $\lambda_{u}$ does not correspond to the one associated to $\lambda_{p}$. It is interesting to underline that, with the new method, the type of 
plastic mechanism associated to the minimum value of $\lambda_{u}$ corresponds to the one appearing in the fully non-linear numerical analysis for $99,5 \%$ of the investigated frames.

As previously mentioned, it is recommended to apply the Merchant-Rankine method to structures with a $\lambda_{p}$ to $\lambda_{c r}$ ratio between 0,1 and 0,25 . If only the frames respecting this condition are considered (which is the case for 150 of the investigated composite structures), the previous observations are still valid:

- Only 13 unsafe situations (i.e. 8,7\% of the considered frames) are obtained with the new method for 57 (i.e. $38 \%$ of the considered frames) with the Merchant-Rankine approach.

- The number of frames for which the difference on the value of $\lambda_{u}$ is between $0 \%$ and $10 \%$ is now equal to 131 with the new method (i.e. $87,3 \%$ of the considered frames) and to 40 with the Merchant-Rankine approach (i.e. 26,7 \% of the considered frames), which confirms the better accuracy of the proposed method.

- The type of plastic mechanism associated to the minimum value of $\lambda_{u}$ obtained with the proposed new method corresponds to the one appearing in the fully non-linear numerical analysis for $100 \%$ of the investigated frames.

In [5], the new method was also applied to actual composite buildings presenting several storeys and bays; it was demonstrated that the new method was able to predict with a good accuracy the ultimate load factor and the associated collapse mode.

\subsection{Simplified method for the computation of $\lambda_{\text {cr }}$}

In the previously mentioned investigations, an accurate value of $\lambda_{c r}$ was computed through the FEM software FINELG and was used within the developed method.

In Eurocode 3, Part 1-1 [11] dedicated to steel structures, a simplified analytical method based on elastic linear analyses is proposed for the computation of this load factor. 
In [12], it is demonstrated that this simplified analytical method can be applied with good confidence to composite frames and that the results obtained through the developed method with this approximated value of $\lambda_{c r}$ are still accurate. Indeed, it is shown that, even if differences of $20 \%$ between the values of $\lambda_{c r}$ analytically predicted and the ones numerically computed are sometimes observed for the steel and composite frames investigated within the parametrical studies, the maximum difference between the $\lambda_{u}$ obtained through the developed method using the different predictions of $\lambda_{c r}$ is equal to $5 \%$.

Accordingly, it can be concluded that the developed method can be applied to a structure using only simple analytical structural analyses.

\section{CONCLUSIONS}

In the past few years, the construction of taller composite buildings and larger composite industrial halls without wind bracing systems made global instability a relevant failure mode, which is not well covered by Eurocode 4 .

Within the present paper, an innovative simplified analytical method aiming at predicting the ultimate load factor and the associated collapse mode of steel and composite sway frames has been presented. This method is in full agreement with Eurocode recommendations and has been validated through parametrical studies. In particular, the very good accuracy of the developed method was demonstrated.

\section{ACKNOWLEDGEMENTS}

The authors would like to address acknowledgements to two collaborators who contributed to the achievement of the presented results, Dr. Ir. Ly Dong Phuong Lam and Ir. Ludivine Comeliau. 


\section{REFERENCES}

[1] EN 1998-1-1, 2004. "Eurocode 8: Design of structures for earthquake resistance - Part 1: General rules, seismic actions and rules for buildings”, European Standard, CEN, Brussels.

[2] Hensman J. S., 1998. "Investigation of the wind-moment method for unbraced composite frames", Ph.D. thesis, Nottingham University (U.K.).

[3] EN 1994-1-1, 2005. "Eurocode 4: Design of Composite Steel and Concrete Structures. Part 1.1: General Rules and Rules for Buildings”, European Standard, CEN, Brussels.

[4] Bitar D., Ryan Y., Caramelli S., Salvatore W., Taucer F., Jaspart J.P., Demonceau J.F., Haller M., Grijalvo J., Heise F.J., Kindmann R., Kraus M., Hoffmeister B., Oppe M. and Stangenberg H., 2006. "Applicability of composite structures to sway frames”, Final report, Report EUR 212913 en, Science Research Development, European Commission.

[5] Demonceau J.F, 2008. "Steel and composite building frames: sway response under conventional loading and development of membrane effects in beams further to an exceptional action", Ph.D thesis, Liège University (Belgium) (freely downloadable at http://orbi.ulg.ac.be/handle/2268/2740).

[6] Demonceau J.F., Hanus F., Jaspart J.P. and Franssen J.M., 2009 « Behaviour of singlesided composite joints at room temperature and in case of fire after an earthquake », International Journal of Steel Structures (IJOSS), Korea, pp. 329-342.

[7] Demonceau J.F., Jaspart J.P., Klinkhammer R., Weynand K., Labory F. and Cajot L.G., 2008. « Recent developments on composite connections », Steel Construction Design and Research Journal, Vol. 1, pp. 71-76. 
[8] Demonceau J.F., 2004. “Applicability of composite structures to sway frames”, Master thesis presented at Liège University (Belgium) (freely downloadable at http://orbi.ulg.ac.be/handle/2268/2739).

[9] Demonceau J.F., Jaspart J.P. and Maquoi R., 2005. "Design of composite sway building frames for global instability", ASCE Journal of Engineering Mechanics, special issue on advances in the stability of framed structures, June 2005, pp. 641-653.

[10] Maquoi R. and Jaspart J.P, 2001. "A simple approach for the design of steel and composite frames accounting for effective overall stability", Festschrift Prof. Richard Greiner, Graz University, Austria.

[11] EN 1993-1-1, 2005. "Eurocode 3: Design of steel structures - Part 1-1: General rules and rules for buildings", European Standard, CEN, Brussels.

[12] Labeye V., 2008. "Contribution to the development of design rules for sway building (in French)", Diploma Work presented at Liège University, academic year 2007-2008. 